\title{
Clinical Implications of High-mobility Group Box-1 (HMGB1) and the Receptor for Advanced Glycation End-products (RAGE) in Cutaneous Malignancy: A Systematic Review
}

\author{
AUSTIN HUY NGUYEN, SHANNON Q. DETTY and DEVENDRA K. AGRAWAL
}

\author{
Department of Clinical and Translational Science, Creighton University School of Medicine, Omaha, NE, U.S.A.
}

\begin{abstract}
Inflammation and the immune system play a role in the development and progression of melanoma, basal cell carcinoma (BCC), and squamous cell carcinoma (SCC). The pro-inflammatory and tumor-promoting effects of the highmobility group box-1 (HMGB1) protein and the receptor for advanced glycation end products (RAGE) have been investigated in these cutaneous malignancies. The clinical implication of these molecules is not fully described. The National Library of Medicine database was searched for articles addressing the clinical relevance of HMGB1 and RAGE in melanoma, BCC, and SCC. This systematic review includes nine articles, with six summarizing RAGE in cutaneous malignancies and three involving HMGB1. RAGE has been found to be up-regulated in SCC lesions, as well as melanoma. Levels of RAGE were highest in stage IV melanomas. Lower levels of soluble RAGE have been associated with poor overall survival in melanoma. Sporadic extracellular expression of HMGBI was evident in BCC and $S C C$ lesions, which could be released by necrotic tumor cells. $H M G B 1$ was found to be a prognostic marker in melanoma, and HMGB1 levels were elevated in patients who were nonresponders to ipilimumab treatment. HMGBI and RAGE could serve as potential prognostic markers or therapeutic targets in treating melanoma, BCC, and SCC, but further research regarding the clinical utility of the HMGB1-RAGE axis in cutaneous malignancies is warranted.
\end{abstract}

This article is freely accessible online.

Correspondence to: D.K. Agrawal, Department of Clinical and Translational Science, Creighton University School of Medicine, 2500 California Plaza, Omaha, NE 68178, U.S.A. Tel: +1 4022802938, Fax: +1 4022801421, e-mail: dkagr@ creighton.edu

Key Words: High-mobility group box 1, HMGB1, receptor for advanced glycation end-products, RAGE, basal cell carcinoma, melanoma, squamous cell carcinoma, review.
Skin cancer is the most common malignancy in North America, when considered as a single entity (1). The various forms of cutaneous malignancies include malignant melanoma, basal cell carcinoma (BCC), and squamous cell carcinoma (SCC). Whereas melanoma has an estimated incidence of 73,870 cases in the United States (2), the yearly incidence of BCCs and SCCs is estimated to be 3.5 million, with BCCs comprising $80 \%$ of these cases (3). Environmental exposure to ultraviolet light is a major risk factor for cutaneous malignancies. However, inflammation and the immune system play a complex and often paradoxical role in cancer development and progression (4). Inflammation is often antitumoral in melanoma, although emerging evidence suggests certain inflammatory pathways to play a critical role in melanomagenesis and tumor progression (5). The immune signatures of both the tumor and host determine melanoma progression, including the tumor microenvironment, tumorinfiltrating lymphocyte profile, and even immune characteristics of regional lymph nodes $(6,7)$. Various immune cells also play fundamental roles in the development and progression of keratinocyte carcinomas. Both immunosuppression and immune T-cell polarization have demonstrated implications in carcinogenesis (8). Additionally, dendritic cell immunophenotype subsets are associated with cutaneous carcinoma prognosis (9). The complex interplay between cutaneous malignancies and the immune system offers wide therapeutic potential. However, this paradigm is not fully understood, necessitating further research into immune signaling molecules and immune function in the context of cutaneous malignancy.

One such immunomodulatory molecule with demonstrated implications in cancer immunobiology is the high-mobility group box 1 (HMGB1) protein. HMGB1 is a highly conserved and ubiquitously expressed protein that has both nuclear and extracellular functions. Nuclear HMGB1 plays a role in DNA replication and repair, recombination, transcription, and genomic stability (10). Extracellular 
HMGB1 is passively released by damaged or necrotic cells, serving as a damage-associated molecular pattern molecule that alerts the innate immune system (10) by binding to tolllike receptor (TLR)-2 and TLR4, or the receptor for advanced glycation end-products (RAGE) (11). HMGB1 is also secreted by activated immune cells and endothelial cells, giving rise to the release of additional pro-inflammatory cytokines and chemokines (10). The ability of HMGB1 to signal cellular stress responses and sustain long-term inflammation may contribute to tumorigenesis and progression (10). The level of HMGB1 is elevated in several malignancies (12), although its exact role in cancer continues to be investigated.

RAGE is a multi-ligand cell-surface receptor of the immunoglobulin superfamily that is considered to be a pattern-recognition receptor (13). RAGE is expressed throughout the body, and binds with advanced glycation endproducts (14), S100 protein family members (15), fibrillar proteins including amyloid- $\beta$ (16), and HMGB1 (17), resulting in the activation of multiple signaling pathways that give rise to downstream pro-inflammatory effects (13). RAGE has been shown to be overexpressed in various malignancies (13), and has been linked to increased proliferation, survival, migration, and invasion of tumor cells in mouse models (18-21).

The HMGB1-RAGE signaling axis has been implicated in numerous malignancies, including head and neck cancer $(16,22)$. While HMGB1 signaling through TLRs is important in cancer immunobiology, the role of TLRs has already been reviewed (23). Other RAGE ligands have also generally been discussed (24). However, the HMGB1 and RAGE interaction appears to have a unique paradigm and independent prognostic value in cancer, as far as we are aware, with no reviews comprehensively discussing the clinical value of these molecules in cutaneous malignancies. There is paucity in the currently available literature regarding the clinical implications of these molecules. In this study, we systematically review the role of HMGB1 and RAGE in human cutaneous tumor biology and discuss their implications in the diagnosis and prognosis of patients.

\section{Materials and Methods}

The National Library of Medicine database was systematically searched through September 2015 using PubMed with the following search terms: melanoma, skin squamous cell carcinoma, or basal cell carcinoma combined with HMGB1 or RAGE. Titles and abstracts were screened for relevance, after which full-texts of articles were retrieved to be reviewed for inclusion. Included articles addressed the clinical value of HMGB1 and RAGE in primary cutaneous malignancies, namely melanoma, basal cell carcinoma, and cutaneous squamous cell carcinoma. Exclusion criteria were: articles not written in English, conference abstracts, and studies not using human subjects or samples.

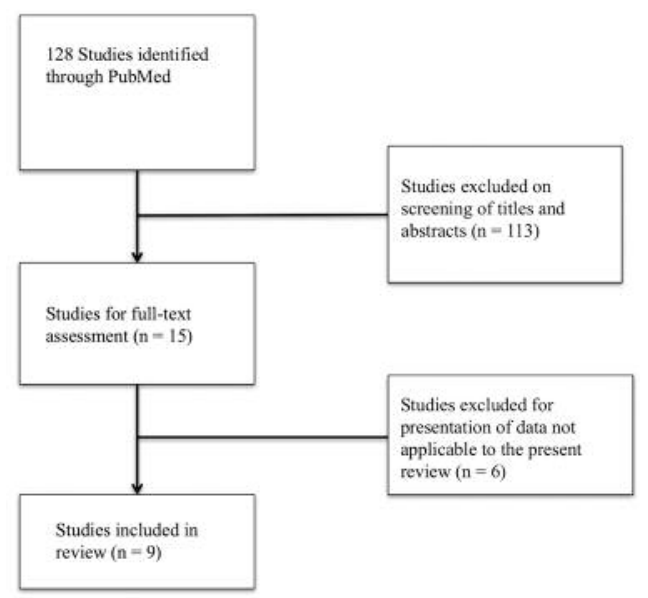

Figure 1. Systematic search of PubMed returned 128 total studies. After review of titles, abstracts, and full text, nine studies were finally included in this review.

\section{Results}

Initial search of the PubMed database returned 128 articles, shown in Figure 1. After screening of titles and abstracts for potential relevance, 15 articles remained for full-text review. Full-text review eliminated six studies due to inappropriate data (use of non-human samples, cell culture only, or animal models) or topic irrelevance to the present review. Ultimately, nine articles were included in this review $(25,26$, 21, 27-32). The majority of the included studies investigated the role of HMGB1 or RAGE in frozen or formalin-fixed paraffin-embedded (FFPE) tissue from patients with melanoma $(25,26,28,29,32)$. Two studies $(30,31)$ used serum from patients with melanoma, and another study (21) used a cDNA array of melanoma cases. Two studies $(27,31)$ assessed tissue from patients with BCC or cutaneous SCC. Characteristics of each study are summarized in Table I.

Melanoma. Investigations of the clinical role of HMGB1 and RAGE in melanoma are summarized in Table II. Li and colleagues (29) found HMGB1 to be highly overexpressed in melanoma tissue compared to controls. Higher levels of HMGB1 were positively correlated with the stage of melanoma, based on the American Joint Committee on Cancer criteria. HMGB1 expression was also increased in cases with increased tumor thickness, increased mitotic index, and presence of lymph node and distant metastasis. HMGB1 expression was a significant prognostic marker for overall and disease-specific 5-year survival. Another study measured serum levels of HMGB1 in patients with metastatic stage IV melanoma receiving ipilimumab treatment. In non-responders, post-therapy serum HMGB1 levels were significantly higher than in patients responding to therapy (30). 
Table I. Summary of study details investigating high-mobility group box-1 and the receptor for advanced glycation end products in cutaneous malignancies.

\begin{tabular}{|c|c|c|c|c|c|c|c|}
\hline Study & Cancer type & $\mathrm{N}$ (total) & $\mathrm{N}$ (cancer) & Gender, M:F & Mean age (range), year & Tissue & Assay \\
\hline Eichmuller et al., 2002 (25) & Melanoma & 10 & 10 & - & - & Frozen & RT-PCR \\
\hline Abe et al., 2004 (26) & Melanoma & 2 & 2 & - & - & FFPE & $\mathrm{IHC}$ \\
\hline Leclerc et al., 2009 (21) & Melanoma & 43 & 40 & $24: 16^{*}$ & $56^{\dagger}(34-81)$ & cDNA array & qPCR \\
\hline Weng et al., 2013 (27) & $\mathrm{BCC}, \mathrm{SCC}$ & 56 & 49 & - & - & FFPE & IHC \\
\hline Zhu et al., 2013 (28) & Melanoma & 70 & 40 & - & - & FFPE & $\mathrm{IHC}$ \\
\hline Li et al., 2014 (29) & Melanoma & 134 & 102 & $60: 42 *$ & - & FFPE & $\mathrm{IHC}$ \\
\hline Gebhardt et al., 2015 (30) & Melanoma & 59 & 59 & $26: 33$ & $65.2^{\dagger}(32-84)$ & Serum & ELISA \\
\hline Iotzova-Weiss et al., 2015 (31) & SCC & 25 & 20 & - & - & FFPE & IHC \\
\hline \multirow[t]{2}{*}{ Wagner et al., 2015 (32) } & Melanoma & 707 & 402 (serum) & $228: 174$ & $60.0(16-88)$ & Serum & ELISA \\
\hline & & & 305 (TMA) & $163: 142$ & $56.6(14-89)$ & TMA & IHC \\
\hline
\end{tabular}

BCC: Basal cell carcinoma, FFPE: formalin-fixed paraffin embedded, ELISA: enzyme-linked immunosorbent assay, IHC: immunohistochemistry, qPCR: quantitative/real-time polymerase chain reaction, RT-PCR: reverse transcription polymerase chain reation, SCC: squamous cell carcinoma, TMA: tissue microarray. *Data excludes controls. ${ }^{\dagger}$ Median.

Table II. Summary of clinical implications of high-mobility group box-1 and the receptor for advanced glycation end products in melanoma.

\begin{tabular}{|c|c|c|}
\hline Study & Marker & Findings \\
\hline Eichmuller et al., 2002 (25) & RAGE & - RAGE gene family members were present in 7/10 metastatic melanoma samples. \\
\hline Abe et al., $2004(26)$ & RAGE & $\begin{array}{l}\text { - RAGE immunoreactivity was detected in the cytoplasm of melanoma cells, } \\
\text { but hardly detected in melanocytes of normal skin. }\end{array}$ \\
\hline Leclerc et al., 2009 (21) & RAGE & $\begin{array}{l}\text { - Stage IV tumors expressed RAGE significantly more than stage III tumors }(p<0.05) \text {. } \\
\text { - In } 90 \% \text { of stage III and stage IV tumors, sRAGE was significantly underexpressed } \\
\text { compared to non-melanoma tissue controls }(p<0.05) \text {. }\end{array}$ \\
\hline Zhu et al., 2013 (28) & RAGE & $\begin{array}{l}\text { - RAGE was expressed in } 95 \%(19 / 20) \text { of primary tumor specimens, with } 16 / 19 \text { having strong } \\
\text { expression. } 100 \%(20 / 20) \text { metastatic tumor specimens strongly expressed RAGE. } \\
\text { Staining pattern was apparently similar in these two groups. } \\
\text { - RAGE immunostaining was faint in nevus controls. }\end{array}$ \\
\hline Li et al., 2014 (29) & HMGB1 & $\begin{array}{l}\text { - Expression was elevated in melanoma cases compared with controls. } \\
\text { - Expression positively correlated with AJCC melanoma staging }(p<0.0001) \text {. } \\
\text { - Higher expression was associated with increased tumor thickness }(p=0.012) \text {, } \\
\text { increased mitotic index }(p<0.0001) \text {, and presence of lymph node and distant } \\
\text { metastasis }(p<0.0001 \text { and } p=0.011 \text {, respectively). } \\
\text { - HMGB1 levels were found to be an independent prognostic factor for overall } \\
\text { (RR=6.14, 95\% CI=2.25 to } 16.76 ; p<0.0001) \text { and disease-specific 5-year survival } \\
(\mathrm{RR}=3.81,95 \% \mathrm{CI}=1.15 \text { to } 12.59 ; p=0.028) \text {. }\end{array}$ \\
\hline Gebhardt et al., 2015 (30) & HMGB1 & $\begin{array}{l}\text { - Serum HMGB } 1 \text { levels in patients not responding to ipilimumab were higher after initial } \\
\text { therapy, when compared to responding patients }(p<0.05) \text {. }\end{array}$ \\
\hline Wagner et al., 2015 (32) & RAGE & $\begin{array}{l}\text { - RAGE was expressed at higher levels in melanoma and stromal cells than in benign nevi }(p<0.001) \text {. } \\
\text { - Serum sRAGE was lower in patients with stage III-IV melanoma when compared to healthy individuals. } \\
\text { - Serum sRAGE was significantly associated with impaired survival }(p=0.034) \text { and was } \\
\text { an independent prognostic marker for impaired overall survival }(p<0.05) \text {. }\end{array}$ \\
\hline
\end{tabular}

AJCC: American Joint Committee on Cancer, CI: confidence interval, HMGB1: high-mobility group box-1, RAGE: receptor for advanced glycation end products, RR: relative risk, sRAGE: soluble receptor for advanced glycation end products.

The remaining investigations of melanocytic cases were focused on RAGE expression. In one study, the majority of metastatic melanoma tissue analyzed by reverse transcription polymerase chain reaction, were found to positively express RAGE (25). Similarly, another study detected elevated
RAGE immunohistochemical reactivity in the cytoplasm of melanoma cells, while normal skin expressed minimal levels (26). Zhu and colleagues found comparable results, with nearly all primary melanoma specimens expressing RAGE, and particularly high levels being expressed in metastatic 
Table III. Summary of clinical implications of high-mobility group box-1 and the receptor for advanced glycation end products in non-melanocytic cutaneous malignancies.

\begin{tabular}{|c|c|c|}
\hline Study & Marker & Findings \\
\hline Weng et al., 2013 (27) & HMGB1 & $\begin{array}{l}\text { - Nuclear expression was diffusely, moderately positive in low malignant BCC and weakly, } \\
\text { diffusely positive in highly malignant SCC. In normal squamous epithelial nuclei, it was strongly, } \\
\text { diffusely positive and significantly different from that of SCC }(p=0.003) \text {. } \\
\text { - Cytoplasmic expression was focally positive in BCC and minimal in SCC. } \\
\text { - Expression in intercellular spaces was sporadic in both BCC and SCC. }\end{array}$ \\
\hline $\begin{array}{l}\text { Iotzova-Weiss et al., } \\
2015 \text { (31) }\end{array}$ & RAGE & $\begin{array}{l}\text { - Protein expression was up-regulated in invasive and in situ SCC of both immunocompetent and } \\
\text { organ transplant recipient patients, when compared to normal skin. } \\
\text { - The epidermal fraction of in situ and invasive SCC tissue from immunocompetent } \\
\text { and organ transplant recipient patients demonstrated increased RAGE mRNA when } \\
\text { compared to normal epidermis (all groups, } p<0.001 \text { ). }\end{array}$ \\
\hline
\end{tabular}

BCC: Basal cell carcinoma, HMGB1: high-mobility group box-1, RAGE: receptor for advanced glycation end products, SCC: squamous cell carcinoma.

tumor specimens. In comparison, immunoreactivity of RAGE in nevus pigmentosus was faint in the benign nevus cells (28).

Leclerc and colleagues surveyed 40 melanoma tumor samples for RAGE transcription and found elevated levels in stage IV when compared to stage III tumors. Conversely, the levels of soluble RAGE (sRAGE) in both stage III and IV tumors were significantly lower than that of nonmalignant controls (21). Separately, a study of serum from a total of 402 patients with melanoma found lower sRAGE levels to be independently and strongly associated with poor overall survival. On the other hand, RAGE protein expression was highly up-regulated in primary melanomas when compared to benign nevi (32).

Non-melanocytic malignancy. Investigation of BCC and SCC was performed in two studies, as summarized in Table III. Weng and colleagues investigated HMGB1 expression in BCC and SCC via immunohistochemistry and western blot analysis. Within epithelial nuclei, there was diffuse, moderate expression of HMGB1 in poorly malignant $\mathrm{BCC}$, and relatively weak diffuse expression in highly malignant SCC, which was significantly lower than that of normal squamous cells. Increased nuclear HMGB1 in normal squamous cells may contribute to stabilization of DNA. Within epithelial cytoplasm, there was focal positive expression of HMGB1 in BCC and minimal expression in SCC. Extracellular HMGB1 expression within epithelial intercellular spaces was sporadic in BCC and SCC, and minimal in normal squamous cells, which could imply that intracellular HMGB1 is released as a result of necrosis of epidermal tumor cells (27).

Investigation of FFPE tissue from patients with SCC found $R A G E$ to be transcriptionally up-regulated in SCC lesions when compared to normal epidermis. RAGE expression was also elevated in organ transplant recipients on long-term immunosuppression when compared to immunocompetent individuals (31).

\section{Discussion}

Overall, patients with cutaneous malignancies exhibit increased intratumoral RAGE expression and decreased serum sRAGE level. The latter demonstrates potential as a prognostic marker for patients with cutaneous melanoma (32). In rodent models, RAGE localization may also be an important prognostic consideration, in addition to the level of expression (33).

An in vitro study suggests that RAGE activation by HMGB1 enhances the protumoral activities of alternative activated type 2 macrophages (34). Furthermore, in hepatocellular carcinoma cell lines, RAGE activation by HMGB1, in turn, activated nuclear factor kappa-light-chainenhancer of activated B-cells, promoting cellular proliferation, invasion, and metastasis (35). Such protumoral effects of RAGE signaling, modeled in Figure 2, make it an attractive therapeutic target. Additionally, considering its involvement in cutaneous cancer biology, $R A G E$ polymorphisms may also possess predictive potential for survival or occurrence of malignancy, although only one negative study (32) has been reported. Downstream effects of HMGB1 and RAGE are generalized to promote inflammation, alter cancer cell survival and metabolism, and modulate immune function, among numerous other effects $(12,24)$. The mechanism by which these modulate tumor cell behavior and cancer progression, particularly in cutaneous lesions, is not fully understood. Should these molecules act similarly to driver mutations, in which downstream constitutive signaling is induced, these molecules would indeed be attractive candidates for targeted molecular therapy. 


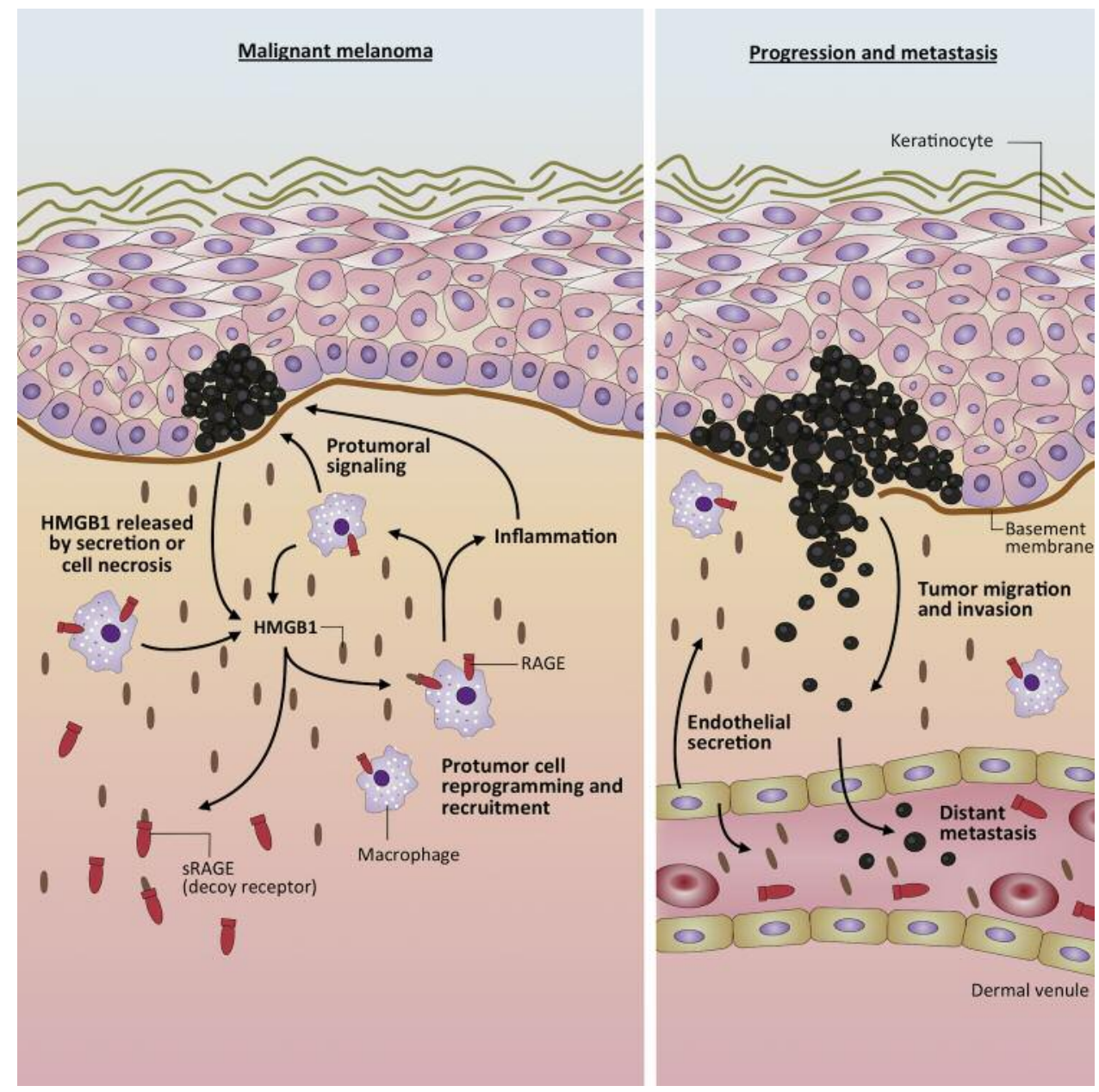

Figure 2. A generalized model of high-mobility group box-1(HMGB1) and the receptor for advanced glycation end products (RAGE) involvement in skin cancer progression. Extracellular HMGB1, released by secretion or cell injury, acts as a proinflammatory cytokine. Interaction with immune cells may reprogram and recruit tumor-associated macrophages that promote tumor progression. Soluble RAGE (sRAGE) acts as a decoy receptor by binding HMGB1 without subsequent signaling.

Specific downstream effects of HMGB1 and RAGE signaling have been elucidated via animal modeling. In a murine SCC model, HMGB1 expression was found to be clearly associated with cancer stage and extent of neoplastic change (36). The level of HMGB1 is clearly increased in melanoma tumors, with higher expression associated with more aggressive lesions (29). In melanoma, HMGB1 serves a trans-regulatory protein that interacts with p65 in the melanoma inhibitory activity promoter, thereby enhancing malignant transformation of melanoma cells (37). Further investigation is required to elucidate the exact role of HMGB 1 in tumor promotion and its potential therapeutic use.

Separately, the role of HMGB 1 has been investigated in cisplatin-treated SCC in a murine model (38). A strong association was found between HMGB1 protein and cisplatin-induced cell death. Gebhardt and colleagues found serum HMGB1 level to be higher in patients with melanoma who did not respond to ipilimumab treatment (30). This establishes the potential for HMGB 1 to be a marker for response to treatment, although further investigation into such application is required.

The available literature establishes a role of HMGB1 and RAGE in cutaneous malignancies, although the current understanding is incomplete. The diagnostic value of these markers in cutaneous malignancies has not been explored; however, the broad effects and roles of these molecules may preclude such a utility. These markers are associated with numerous clinicopathological factors in cutaneous malignancies. This early evidence is promising for clinical use of these molecules in prognostic considerations or as a molecular therapeutic target. 


\section{Financial \& Competing Interests Disclosure}

The Authors have no other relevant affiliations or financial involvement with any organization or entity with financial interest or financial conflict with the subject matter or materials discussed in the manuscript apart from those disclosed. No writing assistance was utilized in the production of this article.

\section{Acknowledgements}

This work was supported by the Department of Clinical and Translational Sciences of Creighton University School of Medicine Omaha, NE, USA.

\section{References}

1 Gandhi SA and Kampp J: Skin Cancer Epidemiology, Detection, and Management. Med Clin North Am 99: 1323-1335, 2015.

2 Siegel RL, Miller KD and Jemal A: Cancer Statistics, 2015. CA Cancer J Clin 65: 5-29, 2015.

3 American Cancer Society: What are basal and squamous cell skin cancers? Key statistics for basal and squamous cell skin cancers. Am Cancer Soc: Accessed May 9, 2016. Available from: http://www.cancer.org/cancer/skincancer-basalandsquamouscell/ detailedguide/skin-cancer-basal-and-squamous-cell-what-isbasal-and-squamous-cell.

4 de Visser KE, Eichten A and Coussens LM: Paradoxical roles of the immune system during cancer development. Nat Rev Cancer 6: 24-37, 2006.

5 Schneider SL, Ross AL and Grichnik JM: Do inflammatory pathways drive melanomagenesis? Exp Dermatol 24: 86-90, 2015.

6 Margolin K: Introduction to the role of the immune system in melanoma. Hematol Oncol Clin North Am 28: 537-558, 2014.

7 Lee N, Zakka LR, Mihm MC and Schatton T: Tumourinfiltrating lymphocytes in melanoma prognosis and cancer immunotherapy. Pathology 48: 177-187, 2016.

8 Yu SH, Bordeaux JS and Baron ED: The immune system and skin cancer. Adv Exp Med Biol 810: 182-191, 2014.

9 Yanofsky VR, Mitsui H, Felsen D and Carucci JA: Understanding dendritic cells and their role in cutaneous carcinoma and cancer immunotherapy. Clin Dev Immunol 2013, 2013.

10 Kang R, Chen R, Zhang Q, Hou W, Wu S, Cao L, Huang J, Yu Y, Fan X-G, Yan Z, Sun X, Wang H, Wang Q, Tsung A, Billiar TR, Zeh HJ, Lotze MT and Tang D: HMGB1 in health and disease. Mol Aspects Med 40: 1-116, 2014.

11 Tang D, Kang R, Zeh HJ and Lotze MT: High-mobility group box 1, oxidative stress, and disease. Antioxid Redox Signal 14: 1315-1335, 2011.

12 Kang R, Zhang Q, Zeh HJ, Lotze MT and Tang D: HMGB1 in cancer: Good, bad, or both? Clin Cancer Res 19: 4046-4057, 2013.

13 Malik P, Chaudhry N, Mittal R and Mukherjee TK: Role of receptor for advanced glycation end products in the complication and progression of various types of cancers. Biochim Biophys Acta 1850: 1898-1904, 2015.

14 Yan SF, Ramasamy R and Schmidt AM: Mechanisms of disease: advanced glycation end-products and their receptor in inflammation and diabetes complications. Nat Clin Pract Endocrinol Metab 4: 285-293, 2008.
15 Leclerc E, Fritz G, Vetter SW and Heizmann CW: Binding of S100 proteins to RAGE: an update. Biochim Biophys Acta 1793: 993-1007, 2009.

16 Sims GP, Rowe DC, Rietdijk ST, Herbst R and Coyle AJ: HMGB1 and RAGE in inflammation and cancer. Annu Rev Immunol 28: 367-388, 2010.

17 Wu X, Mi Y, Yang H, Hu A, Zhang Q and Shang C: The activation of HMGB1 as a progression factor on inflammation response in normal human bronchial epithelial cells through RAGE/JNK/NFKB pathway. Mol Cell Biochem 380: 249-257, 2013.

18 Logsdon CD, Fuentes MK, Huang EH and Arumugam T: RAGE and RAGE ligands in cancer. Curr Mol Med 7: 777-789, 2007.

19 Taguchi A, Blood DC, del Toro G, Canet A, Lee DC, Qu W, Tanji N, Lu Y, Lalla E, Fu C, Hofmann MA, Kislinger T, Ingram M, Lu A, Tanaka H, Hori O, Ogawa S, Stern DM and Schmidt AM: Blockade of RAGE-amphoterin signalling suppresses tumour growth and metastases. Nature 405: 354-360, 2000.

20 Kang R, Tang D, Schapiro NE, Livesey KM, Farkas A, Loughran P, Bierhaus A, Lotze MT and Zeh HJ: The receptor for advanced glycation end products (RAGE) sustains autophagy and limits apoptosis, promoting pancreatic tumor cell survival. Cell Death Differ 17: 666-676, 2010.

21 Leclerc E, Heizmann CW and Vetter SW: RAGE and S100 protein transcription levels are highly variable in human melanoma tumors and cells. Gen Physiol Biophys 28 Spec No: F65-75, 2009.

22 Nguyen A, Bhavsar S, Riley E, Caponetti G and Agrawal D: Clinical value of high mobility group box 1 and the receptor for advanced glycation endproducs in head and neck cancer: A systematic review. Int Arch Otorhinolaryngol 20: 382-389, 2016.

23 Chen K, Huang J, Gong W, Iribarren P, Dunlop NM and Wang JM: Toll-like receptors in inflammation, infection and cancer. Int Immunopharmacol 7: 1271-1285, 2007.

24 Sparvero LJ, Asafu-Adjei D, Kang R, Tang D, Amin N, Im J, Rutledge R, Lin B, Amoscato A a, Zeh HJ and Lotze MT: RAGE (receptor for advanced glycation endproducts), RAGE ligands, and their role in cancer and inflammation. J Transl Med 7: 17, 2009.

25 Eichmüller S, Usener D, Jochim A and Schadendorf D: mRNA expression of tumor-associated antigens in melanoma tissues and cell lines. Exp Dermatol 11: 292-301, 2002.

26 Abe R, Shimizu T, Sugawara H, Watanabe H, Nakamura H, Choei H, Sasaki N, Yamagishi S, Takeuchi M and Shimizu H: Regulation of human melanoma growth and metastasis by AGEAGE receptor interactions. J Invest Dermatol 122: 461-467, 2004.

27 Weng H, Deng Y, Xie Y, Liu H and Gong F: Expression and significance of HMGB1, TLR4 and NF- $\mathrm{kB}$ p65 in human epidermal tumors. BMC Cancer 13: 311, 2013

28 Zhu L, Ito T, Nakahara T, Nagae K, Fuyuno Y, Nakao M, Akahoshi M, Nakagawa R, Tu Y, Uchi $\mathrm{H}$ and Furue M: Upregulation of S100P, receptor for advanced glycation end products and ezrin in malignant melanoma. J Dermatol 40: 9739, 2013.

29 Li Q, Li J, Wen T, Zeng W, Peng C, Yan S, Tan J, Yang K, Liu S, Guo A, Zhang C, Su J, Jiang M, Liu Z, Zhou H and Chen X: Overexpression of HMGB1 in melanoma predicts patient survival and suppression of HMGB 1 induces cell cycle arrest and senescence in association with p21 (WAF1/CIP1) upregulation via a p53-independent, Sp1-dependent pathway. Oncotarget 5: 6387-6403, 2014. 
30 Gebhardt C, Sevko A, Jiang H, Lichtenberger R, Reith M, Tarnanidis K, Holland-Letz T, Umansky L, Beckhove P, Sucker A, Schadendorf D, Utikal J and Umansky V: Myeloid cells and related chronic inflammatory factors as novel predictive markers in melanoma treatment with ipilimumab. Clin Cancer Res, 2015.

31 Iotzova-Weiss G, Dziunycz PJ, Freiberger SN, Läuchli S, Hafner J, Vogl T, French LE and Hofbauer GFL: S100A8/A9 stimulates keratinocyte proliferation in the development of squamous cell carcinoma of the skin Ithe receptor for advanced glycation-end products. PLoS One 10: e0120971, 2015.

32 Wagner NB, Weide B, Reith M, Tarnanidis K, Kehrel C, Lichtenberger R, Pflugfelder A, Herpel E, Eubel J, Ikenberg K, Busch C, Holland-Letz T, Naeher H, Garbe C, Umansky V, Enk A, Utikal $\mathrm{J}$ and Gebhardt C: Diminished levels of the soluble form of RAGE are related to poor survival in malignant melanoma. Int J Cancer 137: 2607-2617, 2015.

33 Todorova $\mathrm{J}$ and Pasheva E: High mobility group B1 protein interacts with its receptor RAGE in tumor cells but not in normal tissues. Oncol Lett 3: 214-218, 2012.

34 Rojas A, Delgado-López F, Perez-Castro R, Gonzalez I, Romero J, Rojas I, Araya P, Añazco C, Morales E and Llanos J: HMGB1 enhances the protumoral activities of M2 macrophages by a RAGE-dependent mechanism. Tumour Biol 37: 3321-3329, 2016.
35 Chen R-C, Yi P-P, Zhou R-R, Xiao M-F, Huang Z-B, Tang D-L, Huang $\mathrm{Y}$ and Fan $\mathrm{X}-\mathrm{G}$ : The role of HMGB1-RAGE axis in migration and invasion of hepatocellular carcinoma cell lines. Mol Cell Biochem 390: 271-280, 2014.

36 Sharma A, Ray R and Rajeswari MR: Overexpression of high mobility group (HMG) B1 and B2 proteins directly correlates with the progression of squamous cell carcinoma in skin. Cancer Invest 26: 843-851, 2008.

37 Poser I, Golob M, Buettner R and Bosserhoff AK: Up-regulation of $H M G 1$ leads to melanoma inhibitory activity expression in malignant melanoma cells and contributes to their malignancy phenotype. Mol Cell Biol 23: 2991-2998, 2003.

38 Sharma A, Ramanjaneyulu A, Ray R and Rajeswari MR: Involvement of high mobility group B proteins in cisplatininduced cytotoxicity in squamous cell carcinoma of skin. DNA Cell Biol 28: 311-318, 2009.
Received November 5, 2016

Revised November 28, 2016

Accepted November 29, 2016 